\title{
Terahertz wave imaging: horizons and hurdles
}

\author{
X-C Zhang \\ Center for Terahertz Research, Rensselaer Polytechnic Institute, Troy, NY 12180-3590, USA \\ E-mail: zhangxc@rpi.edu
}

Received 18 March 2002

Published 17 October 2002

Online at stacks.iop.org/PMB/47/3667

\begin{abstract}
Terahertz $(\mathrm{THz})$ science will profoundly impact biotechnology. It has tremendous potential for applications in imaging, medical diagnosis, health monitoring, environmental control and chemical and biological identification. $\mathrm{THz}$ research will become one of the most promising research areas in the 21 st century for transformational advances in imaging, as well as in other interdisciplinary fields. However, terahertz wave (T-ray) imaging is still in its infancy. This paper discusses the uniqueness and limitations of T-ray imaging, identifies the major challenges impeding T-ray imaging and proposes solutions and opportunities in this field. It also concentrates on the generation, propagation and detection of T-rays by the use of femtosecond optics.
\end{abstract}

\section{Introduction}

Terahertz (THz) waves, with a frequency range of 0.1-10 THz, are termed 'T-rays'. They occupy a large portion of the electromagnetic spectrum between the infrared and microwave bands. Compared to the relatively well-developed science and technology at microwave, optical and X-ray frequencies, basic research, new initiatives and advanced technology developments in the $\mathrm{THz}$ band are very limited and remain relatively unexplored.

The large terahertz portion of the spectrum has not been well developed because there were neither convenient high-power emitters to send out controlled terahertz signals nor efficient sensors to collect them and record information. Developments in THz time-domain spectroscopy and related $\mathrm{THz}$ technologies now lead us to view the world in a new way. As a result of developing research, terahertz radiation now has widespread potential applications in medicine, microelectronics, agriculture, forensic science and many other fields.

During the last 15 years, the fundamentals of free-space $\mathrm{THz}$ optoelectronics have seen significant advances with the development of solid state mode-locked lasers (especially Ti:sapphire lasers) and microelectronics fabrication (such as micron size planar photoconductive antennas). Several different optoelectronic techniques for the generation, propagation and detection of T-rays have been developed. Free-space pulsed $\mathrm{THz}$ optoelectronic radiation and detection systems, which use photoconductive antennas or 
electro-optic crystals, provide diffraction-limited spatial resolution, femtosecond temporal resolution, dc-THz spectral bandwidth and sub- $\mathrm{mV} \mathrm{cm}^{-1}$ field sensitivity.

$\mathrm{THz}$ time-domain spectroscopy and imaging has been applied to material science, physics, electrical engineering and chemistry. Potential applications in biology and medicine are now beginning to emerge. In the same way that visible light can create a photograph, radio waves can transmit sound and x-rays can view within the human body, terahertz waves can create images and transmit information.

Several properties of $\mathrm{THz}$ wave radiation triggered research to develop this frequency band for biomedical applications. T-rays have several advantages over x-rays, one being that they have low photon energies (for example, $4 \mathrm{meV}$ at $1 \mathrm{THz}$ ) and therefore do not subject a biological tissue to harmful radiation [1]. In comparison, typical x-ray photon energy is in the range of $\mathrm{keV}$, which is 1 million times higher than that of a T-ray photon. While microwave and $\mathrm{x}$-ray imaging modalities produce density pictures, T-ray imaging also provides spectroscopic information within the terahertz frequency range. The unique rotational, vibrational and translational responses of materials (molecular, radicals and ions) within the $\mathrm{THz}$ range provide information that is generally absent in optical, x-ray and NMR images. In principle, these transitions in $\mathrm{THz}$ frequency are specific to the molecule and therefore enable $\mathrm{THz}$ wave fingerprinting. This conformational information is closely related to biological functions of the molecules in tissues and cells and is difficult to access with other techniques.

Coherent $\mathrm{THz}$ wave signals are detected in the time domain by mapping the transient of the electric field in amplitude and phase. This gives access to absorption and dispersion spectroscopy. T-rays can also easily penetrate and image inside most dielectric materials, which may be opaque to visible light and low contrast to X-rays, making T-rays a useful and complementary imaging source in this context.

A goal of T-ray imaging is to produce images with 'component contrast' enabling an analysis of the water content and composition of tissues in biological samples. Such a capability presents tremendous potential to identify early changes in composition and function as a precursor to specific medical investigations and treatment. Moreover, in conventional optical transillumination techniques that use near-infrared pulses, large amounts of scattering can spatially smear out the objects to be imaged. T-ray imaging techniques, due to their longer wavelengths, can provide significantly enhanced contrast because of low scattering (Rayleigh scattering).

Since the onset of imaging with T-rays, in 1995 [2], various T-ray imaging modalities for numerous applications have been proposed, developed and demonstrated [3-37]. They include electro-optic imaging [3], tomographic imaging [6], single-shot imaging [9], near-field imaging [15, 20], dark-field imaging [26], bistatic THz imaging [32, 33] and T-ray computed tomography (CT) [36, 37].

This paper discusses recent developments in T-ray imaging and reviews the major hurdles to be overcome towards the goal of a practical biomedical application. Sections 2 and 3 briefly review terahertz history and state-of-the-art techniques in pulsed $\mathrm{THz}$ generation and detection with emphasis on optoelectronic techniques. Section 4 offers a vision of the future by describing a futuristic medical $\mathrm{THz}$ imaging system that would fill a pressing need in the medical imaging community. This vision is used as the motivation for a discussion in section 5 on the prominent challenges facing $\mathrm{THz}$ imaging and potential solutions.

\section{Generation of terahertz waves}

Currently, there are two basic methods for generating T-rays using ultra-fast laser pulses: photoconduction and optical rectification $[38,39]$. A typical optical source for the generation 
of T-rays is an ultra-fast Ti:sapphire laser with an average power of nearly $1 \mathrm{~W}$, a pulse duration of $100 \mathrm{fs}$ and a centre wavelength at $800 \mathrm{~nm}$. The photoconductive approach employs high-speed photoconductors as transient current sources for radiating antennas. The optical rectification approach uses electro-optic crystals as rectification media. Rectification can be a second order (difference frequency generation) [40-45] or higher order non-linear optical process [46], depending on the optical power density.

Optical rectification is the inverse process of the electro-optic effect [40]. In contrast to photoconducting elements where the optical beam functions as a trigger, the energy of $\mathrm{THz}$ wave radiation, generated by the transient optical rectification process, comes from the excitation laser pulse. The conversion efficiency $\left(10^{-4}\right.$ to $\left.10^{-6}\right)$ depends on the value of the non-linear coefficient and the phase-matching condition [47-50]. In the optical rectification mode, the $\mathrm{THz}$ pulse duration is comparable to the optical pulse duration, and the frequency spectrum is mainly limited by the bandwidth of the laser pulse.

In general, photoconductive antennas provide higher T-ray power than the use of optical rectification. This is due to the fact that the T-ray energy is pre-stored in the antenna structure as dc coupled electrostatic energy. The pulsed optical excitation triggers the switch to release the energy as radiation. In theory, the total T-ray power can be higher than the input triggering optical energy. However, optical rectification converts part of the incident optical energy into $\mathrm{THz}$ radiation, so the total T-ray power is much smaller than that of the optical excitation beam. The typical conversion efficiency of optical power to T-ray power ranges from $10^{-3}$ to $10^{-6}$.

\section{Detection of terahertz waves}

In a similar fashion to the generation of T-rays, both photoconductive and electro-optic methods can be used to detect T-rays. Historically, a photoconductive antenna was first used to detect freely propagating T-rays [38]. Electro-optic detection, an alternative method demonstrated 7 years ago [51-58], has become widely used in many research laboratories due to its ultra-wide bandwidth and parallel imaging capability.

In an electro-optic sampling setup, the field-induced birefringence of the sensor crystal, due to an applied electric field ( $\mathrm{THz}$ wave), will modulate the polarization ellipticity of an optical probe beam that passes through the crystal. The ellipticity modulation of the optical beam can then be polarization analysed to provide information on both the amplitude and the phase of the applied electric field. A balanced detection system analyses the polarization change from the electro-optic crystal and correlates it with the amplitude and phase of the $\mathrm{THz}$ electric field. The time evolution of the T-ray waveform is provided by changing the relative length of the beam path between the THz radiation pulses and the optical probe pulses (pumpprobe sampling method). Detection sensitivity is significantly improved by increasing the interaction length of the pulsed field and optical probe beam within the crystal, accomplished by using a thicker crystal. As a quasi-optical beam, a T-ray can be reflected and collimated by metallic mirrors and focused by a plastic or high-resistivity silicon lens. In a T-ray sensing measurement, a target sample is placed within the $\mathrm{THz}$ beam path and the transmitted and/or reflected $\mathrm{THz}$ waveform (phase and amplitude) contains information about the interaction between the T-rays and the sample.

\section{A vision of biomedical imaging}

In this section one particular goal of $\mathrm{THz}$ imaging is presented. This vision is certainly not the only possible biomedical application of $\mathrm{THz}$ imaging and is not necessarily achievable 
in the short to mid term. It is provided to elucidate the strengths of THz imaging and how they might find application in a medical context. It is also used to provide a focus for the subsequent section, which discusses some of the major hurdles impeding this goal. This vision combines the capabilities of a number of current research directions including near-field T-ray microscopes, tomographic reconstruction and endoscopic techniques. We discuss the applications of a three-dimensional (3D) $\mathrm{THz}$ molecular imaging system and briefly review the competing technologies.

In vivo molecular imaging is considered as the next frontier in medical diagnostics. In the ideal situation, this imaging would be performed non-invasively. However, it is difficult to conceive of such a system, instead we will consider an endoscopic $\mathrm{THz}$ imaging probe capable of near-field micrometer resolution and spectroscopic analysis. The development of such a tool would be an entirely new technology that would provide potential for earlier detection and characterization of disease, understanding of biology and evaluation of treatment.

Physicians currently rely on relatively gross parameters of disease. In the case of cancer, this typically includes tumour burden, anatomic location and similar parameters. Disease characterization may be improved by using more specific parameters, such as the detection of pre-malignant molecular abnormalities, growth kinetics, angiogenesis growth factors, tumour cell markers or genetic alterations.

This technology would also allow for the assessment of therapeutic effectiveness at a molecular level, long before phenotypic changes occur. Molecular in vivo imaging may allow for the study of pathogenesis within intact microenvironments of living systems. From the current clinical perspective, the T-ray microscope has tremendous potential. We envision a tool featuring a sampling probe that would be placed within an organ through the skin. Once the probe was in place, an in vivo image would be obtained consisting of a tomographic image and a multispectral analysis of a 3D volume of tissue. A picture of a tumour, for example, would be reconstructed using multiple T-ray images at different angles. This method creates a biologically accurate $3 \mathrm{D}$ picture that gives a researcher a better understanding of the disease and how it has spread.

One of the breakthrough elements of sampling molecular information is the development of an imaging system capable of high spatial resolution and sensitivity. The most commonly used imaging techniques for extraction of molecular information are positron emission tomography (PET), magnetic resonance (MR) and optical techniques. PET is frequently used when a substrate to a given target exists that can only be labelled to a positron emitter. PET has high sensitivity: however, its spatial resolution is poor. MR imaging has two potential advantages over PET. It has a higher spatial resolution (micrometre compared to several millimetres) and the fact that physiologic and anatomic detail can be extracted simultaneously.

In comparison with PET imaging, MR imaging is several magnitudes less sensitive (millimolar rather than picomolar), which is why reliable signal amplification strategies must be developed. Recently, cell labelling techniques have been developed that will allow efficient in vivo tracking of cell lines expressing transgenes, potentially at the single cell level.

\section{Challenges and hurdles}

Sensing and imaging with terahertz frequency for biomedical applications faces many challenges [59]. Various factors severely constrain plausible scenarios for the application of terahertz technology. This section discusses the challenges facing $\mathrm{THz}$ wave imaging. These challenges, including signal-to-noise ratio (SNR), acquisition rate and resolution, reflect common problems encountered in a number of imaging modalities. Other challenges, such 
as the need for a spectroscopic database for biological tissues, are unique to $\mathrm{THz}$ imaging. Where appropriate, recent progress addressing these problems is highlighted and potential future research directions are described.

\subsection{Water}

Perhaps the most restrictive challenge facing $\mathrm{THz}$ imaging in biomedical applications is the high absorption rate of water and other polar liquids. The absorption coefficient for liquid water is as high as $150 \mathrm{~cm}^{-1}$ at $1 \mathrm{THz}$. This strong absorption limits the sensing and imaging in water-rich samples for most terahertz applications and prohibits transmission-mode imaging through a thick tissue. For this reason, current biomedical $\mathrm{THz}$ research has primarily focused on skin conditions $[26,31]$.

\subsection{Power}

The typical average power of the $\mathrm{THz}$ wave source is of the order of $\mu \mathrm{W}$ range (from $0.1 \mu \mathrm{W}$ to $100 \mu \mathrm{W}$ ) with the use of a pulsed laser oscillator at $1 \mathrm{~W}$ output power. The peak power of a $\mathrm{THz}$ wave ranges from $100 \mu \mathrm{W}$ to $0.1 \mathrm{~W}$ depending on the duty cycle of the oscillator. For sensing application with single pixel detection, this power can provide a signal-to-noise ratio of $10^{4}$ or higher. However, for a detector array system for real-time $2 \mathrm{D}$ imaging, an average of $\mathrm{mW}$ or higher (or a peak power of $\mathrm{kW}$ ) is preferred. This may require an amplified spatial resolution.

The resolution of conventional T-ray imaging systems is limited by the wavelength of the $\mathrm{THz}$ radiation $(0.3 \mathrm{~mm}$ for $1 \mathrm{THz})$. This is not detailed enough for a number of applications including imaging of cellular structures. There is, therefore, widespread interest in techniques to improve the spatial resolution of T-ray imaging.

Near-field imaging can greatly improve the spatial resolution of T-ray sensing and imaging systems. Initial experiments demonstrated $110 \mu \mathrm{m}$ and $232 \mu \mathrm{m}$ spatial resolution for $\lambda=$ $125 \mu \mathrm{m}$ and $\lambda=1 \mathrm{~mm}$, respectively [61]. Other groups then used a collection mode near-field imaging technique utilizing a small aperture in a metallic film to block all but a small fraction of the $\mathrm{THz}$ radiation. The resolution is determined by the size of the aperture, but is limited by the thickness of the metallic film, which must be thick enough to prevent leakage of $\mathrm{THz}$ radiation through the film. A resolution of $7 \mu \mathrm{m}$ has been demonstrated using this technique $[20,28]$. The limitation of such a system is the extremely low throughput of the T-rays past the emitter tip, since the transmitted T-ray field is inversely proportional to the third power of the aperture size. It is nearly impossible to obtain a sub-micron spatial resolution with the present T-ray generation and detection technologies.

Another technique for near-field imaging utilizes a dynamic aperture [15, 22]. A THz beam is focused on a semiconductor wafer ( $\mathrm{GaAs}$ or $\mathrm{Si}$ as the gating material). An optical pulse, which is synchronized with an optical pump and a probe beam, is focused at the centre of the THz beam spot. The optical gating pulse creates a conducting layer at the focal point by photo-induced free carriers; the layer then modulates the transmitted $\mathrm{THz}$ beam. The spatial resolution of this method is determined by the focus size of the near-infrared laser beam and a resolution of $(\lambda / 100)$ has been demonstrated. One drawback of this method is the difficulty in coating a gating material on the surface of biomedical samples such as cells and tissues.

Another potential drawback of near-field techniques is the requirement to scan the target. This results in prohibitive acquisition times. A near-field CCD imaging technique would require advanced algorithms to deal with the problems of diffraction and has not yet been considered in the literature. 


\subsection{Signal-to-noise ratio}

$\mathrm{THz}$ time-domain spectroscopy systems are capable of very high signal-to-noise ratios (SNRs) of over 100000 . However, in biomedical imaging, a number of factors combine to dramatically reduce the SNR to the point where it is a limiting concern. Some of these factors include the need to accelerate the imaging acquisition speed (see section 5.4) and the high absorption of biological tissue (section 5.1).

Solutions to the problem of SNR are sought in improving the T-ray hardware. $\mathrm{THz}$ sources have very low average output powers and $\mathrm{THz}$ sensors have relatively low sensitivity compared to sources and sensors operating in the optical spectrum. Both of these aspects of T-ray systems are foci of current research and continue to improve. Other problems are related to the $\mathrm{THz}$ generation process, which results in $\mathrm{THz}$ beams that are not Gaussian and cannot be collimated as well as optical beams. This results in additional noise in $\mathrm{THz}$ images. Potential solutions to the SNR problem may be found in free-electron lasers or in all electronic THz systems [61] although currently each of these alternatives has its own disadvantages.

\subsection{Acquisition speed}

Conventional $\mathrm{THz}$ imaging systems rely on scanning the sample in $x$ and $y$ dimensions to obtain an image. This places severe limits on the available acquisition speed. The first system [2] demonstrated an acquisition rate of 12 pixels/s. Recently, a 50 pixels/s rate has been demonstrated [34] but significant advances are required to allow real-time imaging. Two-dimensional electro-optic sampling has been used together with a CCD camera to provide a dramatic increase in imaging speed [3] and rates as high as 5000 pixels/s are feasible. However, the relegation of the lock-in amplifier results in a significant reduction in SNR compared to the scanned approach. The use of a chirped probe pulse to allow simultaneous sampling of the THz temporal profile [7, 9] can provide a comparable imaging speed to 2D electro-optic sampling, but in addition to a reduced SNR this technique has the additional disadvantages of reduced frequency bandwidth and a limited temporal window. Progress in this domain is largely reliant on other technologies, and improvements are expected to arise from developments such as faster galvanometric stages and lock-in CCD cameras.

\subsection{Limited frequency bandwidth}

Currently, standard photoconductive dipole antenna (PDA) $\mathrm{THz}$ sources are limited to frequencies below 3 or $4 \mathrm{THz}$. Optical rectification provides a wider bandwidth and generation and detection bandwidths in excess of $30 \mathrm{THz}$ have been demonstrated [62, 63], however this is at the expense of $\mathrm{THz}$ power (and therefore SNR). Ideally, a THz imaging system would allow tissue responses to be measured up into the infrared. This would not only allow broader signatures to be observed but also allow the potential for reduced water attenuation, which falls dramatically as the frequency increases over $100 \mathrm{THz}$.

Optical parametric generation of a $\mathrm{CW} \mathrm{THz} \mathrm{wave} \mathrm{provides} \mathrm{a} \mathrm{tunable,} \mathrm{narrow} \mathrm{bandwidth}$ radiation source. With a seed idler beam from a laser diode $(1.07 \mu \mathrm{m})$, a YAG laser at $10.6 \mu \mathrm{m}$ generates a $\mathrm{THz}$ wave in a $\mathrm{LiNbO}_{3}$ crystal in a phase-match condition [64]. The $\mathrm{THz}$ wavelength can be tuned from $0.7 \mathrm{THz}$ to $2.4 \mathrm{THz}$, and the bandwidth is less than $2 \mathrm{MHz}$ (instrument limitation). Other $\mathrm{CW} \mathrm{THz} \mathrm{sources,} \mathrm{using} \mathrm{optical} \mathrm{down} \mathrm{conversion,} \mathrm{include} \mathrm{the}$ beating frequency from two semiconductor diode lasers on a photomixer; this will provide a low cost, tunable THz source with very narrow bandwidth [65]. 


\subsection{Scattering}

Scattering is a common problem for many imaging modalities. In x-ray tomography, scattering of $\mathrm{x}$-ray photons causes artefacts in reconstruction, while in optical tomography of human tissue, scattering is the main transport phenomenon and algorithms are based on modelling photon propagation as a diffusive process. T-rays exhibit significantly reduced scattering in human tissue compared to near-infrared optical frequencies due to the increased wavelength. However, scattering remains an important concern because it is neglected by common processing algorithms. Recently, the scattering of $\mathrm{THz}$ radiation has been investigated using Teflon spheres and scattering related dispersion was noted [66]. This may allow the scattering process to be accurately modelled to aid the future development of diffusion imaging algorithms, such as those adopted for near-infrared imaging. Other authors have compared the scattered and ballistic $\mathrm{THz}$ radiation to yield additional information concerning the sample under study and have shown that this technique has promise with regard to cancer detection [26].

\subsection{Target reconstruction}

Much of the literature concerning T-ray characterization of materials considers only transmission through thin parallel-faced samples, or reflection from relatively flat surfaces $[6,67,68]$. However, a large class of applications calls for imaging of irregularly shaped 3D objects. This presents a number of difficulties in terms of collection optics and reconstruction algorithms. Recently, several groups have focused their attention on this problem resulting in a number of techniques and algorithms for target reconstruction. A synthetic aperture radarbased technique has been demonstrated [27] whereby reflection-mode images of the target are obtained at multiple angles and the 3D reflecting profile of the target is reconstructed. In addition, a biostatic $\mathrm{THz}$ imaging system consisting of $\mathrm{THz}$ receivers at multiple angles relative to the illuminating antenna has been used to image cylindrical reflecting structures [32] and irregular apertures [30].

Another recent development addressing this question is T-ray computed tomography (T-ray CT) [35]. By using this technique, a transmission-mode image of the target is obtained at multiple projection angles. The filtered back-projection algorithm is then used to reconstruct the far-infrared optical properties of the 3D structure. It has been shown that this technique is capable of performing the reconstruction at multiple frequencies and thus extracting spectroscopic information from the target [36]. Much of future research in this domain will focus on improving algorithms to allow reconstruction of complex targets in the presence of noise.

The applicability of T-ray tomography is limited by the reconstruction algorithms. The current simple algorithm does not describe the full interaction of $\mathrm{THz}$ radiation with complex structures and more sophisticated methods are required before strongly diffracting objects can be imaged accurately. The resolution of the T-ray CT is about $4 \mathrm{~mm}$. Better algorithms will further improve the spatial resolution. A parallel development of T-ray CT is T-ray diffraction tomography (DT). This diffraction tomography system provides a speed increase of an order of magnitude over previous T-ray computed tomography systems and allows diffraction effects to be observed and utilized during reconstruction [69]. T-ray DT may potentially provide $0.1 \mathrm{~mm}$ spatial resolution.

\subsection{Biomedical spectroscopic database}

One of the primary advantages of THz imaging over competing techniques is the availability of spectroscopic data within a potentially crucial frequency band. Unfortunately, the responses 
of many biological tissues are unknown in this band. While work to characterize tissues has commenced, such as DNA [1, 46, 47], a significant amount of research remains to be done. This problem is compounded by the fact that there are an enormous number of intraand inter-molecular interactions that have an impact within this frequency regime, making interpretation of the detected spectra very difficult.

An associated problem is the development of computer aided diagnostic algorithms for interpreting the multispectral images obtained by T-ray imaging. A number of authors have considered this question by fitting the measured data to linear filter models and using the filter coefficients as a means to classify gas mixtures [5] and tissue types [21]. One of the most important potential applications for terahertz technology is the detection and identification of biological and chemical agents [70-73].

\subsection{Size}

Current T-ray imaging systems require areas of a few square metres, most of which is dominated by the ultra-fast laser. The goal of an endoscopic T-ray probe requires a number of significant advances. One recent enabling technology is that of the T-ray transceiver [23]. This technique utilizes the reciprocal relationship between optical rectification and electro-optic detection to allow a single $\langle 110\rangle$ ZnTe crystal for both emission and detection of THz pulses. In principle, such a transceiver could be made as small as $1 \mathrm{~mm}^{2}$ and mounted at the end of an optical fibre for endoscopic applications. A large number of practical issues remain unresolved, not the least of which is the problem of dispersion of the ultra-fast pulse.

\subsection{Cost}

Finally, it is worth noting that the high cost of ultra-fast Ti:sapphire lasers impedes $\mathrm{THz}$ imaging in a number of application settings. The typical cost of a T-ray sensing system and an imaging system is $\$ 100000$ and $\$ 200000$, respectively. This price may be acceptable for academic research, but may be too high for general medical applications. Nevertheless, T-ray systems compare favourably in price with X-ray CT and NMR systems. All solid state electronic T-ray sources may greatly reduce the total cost.

\section{Conclusion}

There is no doubt that $\mathrm{THz}$ wave imaging is an attractive technique with enormous potential in biomedical applications. It has a number of important advantages over competing techniques that may give rise to a number of niche applications. To date, however, it has seen only limited exposure in imaging teeth and skin cancers such as basal cell carcinoma. A number of technological challenges are inhibiting more widespread use. We have discussed a number of the most pressing challenges and highlighted potential avenues for their resolution.

\section{Acknowledgments}

This work was supported by the US Army Research Office and the US National Science Foundation. We thank Brad Ferguson for his contribution to this paper.

\section{References}

[1] Smye S W, Chamberlain J M, Fitzgerald A J and Berry E 2001 The interaction between terahertz radiation and biological tissue Phys. Med. Biol. 46 R101-R112 
[2] Hu B B and Nuss M C 1995 Imaging with terahertz waves Opt. Lett. 20 1716-8

[3] Wu Q, Hewitt T D and Zhang X-C 1996 Two-dimensional electro-optic imaging of terahertz beams Appl. Phys. Lett. 69 1026-8

[4] Wu Q, Sun F G, Campbell P and Zhang X-C 1996 Dynamic range of an electro-optic field sensor and its imaging applications Appl. Phys. Lett. 68 3224-6

[5] Mittleman D M, Jacobson R H and Nuss M C 1996 T-ray imaging IEEE J. Sel. Top. Quantum Electron. 2 679-92

[6] Mittleman D M, Hunsche S, Boivin L and Nuss M C 1997 T-ray tomography Opt. Lett. 22 904-6

[7] Jiang Z and Zhang X-C 1998 Free-space electro-optic sampling of THz radiation with chirped optical beam Ultrafast Phenomena XI vol 63 (Berlin: Springer) p 197

[8] Hunsche S, Koch M, Brener I and Nuss M C 1998 THz near-field imaging Opt. Commun. 150 22-6

[9] Jiang Z and Zhang X-C 1998 Single-shot spatiotemporal terahertz field imaging Opt. Lett. 23 1114-6

[10] Arnone D D, Ciesla C M, Corchia A, Egusa S, Pepper M, Chamberlain J M, Bezant C, Linfield E H, Clothier R and Khammo N 1999 SPIE: Applications of terahertz (THz) technology to medical imaging Terahertz Spectroscopy and Applications (Munich, Germany) vol 3828 pp 209-19

[11] Chen Q, Jiang Z and Zhang X-C 1999 All-optical THz imaging Proc. SPIE 3617 98-105

[12] Jiang Z and Zhang X-C 1999 Terahertz imaging via electro-optic effect IEEE Trans. Microw. Theor. Tech. 47 2644-50

[13] Koch M 1999 THz imaging: fundamentals and biological applications Proc. SPIE-Conf. on Terahertz Spectroscopy and Applications (Munich, Germany) vol 3828 pp 202-8

[14] Mittleman D M, Gupta M, Neelamani R, Baraniuk R G, Rudd J V and Koch M 1999 Recent advances in terahertz imaging Appl. Phys. B 68 1085-94

[15] Chen Q, Jiang Z, Xu G X and Zhang X-C 2000 Near-field terahertz imaging with a dynamic aperture Opt. Lett. $251122-4$

[16] Han P Y, Cho G C and Zhang X-C 2000 Time-domain transillumination of biological tissues with terahertz pulses Opt. Lett. $25242-4$

[17] Jiang Z, Xu X G and Zhang X-C 2000 Improvement of terahertz imaging with a dynamic subtraction technique Appl. Opt. $392982-7$

[18] Jiang Z and Zhang X-C 2000 Measurement of spatio-temporal terahertz field distribution by using chirped pulse technology IEEE J. Quantum Electron. 36 1214-22

[19] Mickan S, Abbott D, Munch J, Zhang X C and van Doorn T 2000 Analysis of system trade-offs for terahertz imaging Microelectron. J. 31 503-14

[20] Mitrofanov O, Brener I, Harel R, Wynn J D, Pfeiffer L N, West K W and Federici J 2000 Terahertz near-field microscopy based on a collection mode detector Appl. Phys. Lett. 77 3496-8

[21] Ferguson B, Wang S, Gray D, Abbott D and Zhang X-C 2001 Terahertz imaging of biological tissue using a chirped probe pulse Proc. SPIE: Electronics and Structures for MEMS II (Adelaide, Australia) vol 4591, ed N W Bergmann pp 172-84

[22] Chen Q and Zhang X-C 2001 Semiconductor dynamic aperture for near-field terahertz wave imaging IEEE J. Sel. Top. Quantum Electron. 7 608-14

[23] Chen Q, Tani M, Jiang Z and Zhang X C 2001 Electro-optic transceivers for terahertz-wave applications J. Opt. Soc. Am. B 18 823-31

[24] Johnson J L, Dorney T D and Mittleman D M 2001 Interferometric imaging with terahertz pulses IEEE J. Sel. Top. Quantum Electron. 7 592-9

[25] Johnson J L, Dorney T D and Mittleman D M 2001 Enhanced depth resolution in terahertz imaging using phase-shift interferometry Appl. Phys. Lett. 78 835-7

[26] Loffler T, Bauer T, Siebert K J, Roskos H G, Fitzgerald A and Czasch S 2001 Terahertz dark-field imaging of biomedical tissue Opt. Exp. 9616

[27] McClatchey K, Reiten M T and Cheville R A 2001 Time resolved synthetic aperture terahertz impulse imaging Appl. Phys. Lett. 79 4485-7

[28] Mitrofanov O, Lee M, Hsu J W P, Brener I, Harel R, Federici J F, Wynn J D, Pfeiffer L N and West K W 2001 Collection-mode near-field imaging with 0.5-THz pulses IEEE J. Sel. Top. Quantum Electron. 7 600-7

[29] O'Hara J and Grischkowsky D 2001 Quasi-optic terahertz imaging Opt. Lett. 26 1918-20

[30] Ruffin A B, Decker J, Sanchez-Palencia L, Le Hors L, Whitaker J F, Norris T B and Rudd J V 2001 Time reversal and object reconstruction with single-cycle pulses Opt. Lett. 26 681-3

[31] Woodward R M, Cole B, Walace V P, Arnone D D, Pye R, Linfield E H, Pepper M and Davies A G 2001 Terahertz pulse imaging of in vitro basal cell carcinoma samples SPIE: Conf. on Lasers and Electro-optics 2001 pp 329-30

[32] Dorney T D, Johnson J L, Rudd J V, Baraniuk R G, Symes W W and Mittleman D M 2001 Terahertz reflection imaging using Kirchoff migration Opt. Lett. 261513 
[33] Planken P 2001 A terahertz system using semi-large emitters: noise and performance characteristics Proc. Biomedical Imaging and Sensing Applications of Terahertz Technology (Leeds, UK)

[34] Herrmann M, Tani M, Sakai K and Fukasawa R 2002 Terahertz imaging of silicon wafers J. Appl. Phys. 91 1247-50

[35] Ferguson B, Wang S H and Zhang X-C 2001 T-ray computed tomography Post deadline Paper, PD1.7 IEEE/LEOS Annual Meeting (San Jose, CA)

[36] Ferguson B, Wang S H, Gray D, Abbott D and Zhang X-C 2002 Towards functional 3D T-ray imaging Phys. Med. Biol. 47 3735-42

[37] Ferguson B, Wang S H, Gray D, Abbott D and Zhang X-C 2002 T-ray computed tomography Opt. Lett. 271312

[38] Auston D H and Smith P R 1983 Generation and detection of millimeter waves by picosecond photoconductivity Appl. Phys. Lett. 43 631-3

[39] Zhang X-C, Jin Y, Hewitt T D, Kingsley L E and Weiner M 1993 THz radiation by carrier transport or optical rectification Ultrafast Electron. Optoelectron. 1499

[40] Bass M, Franken P A, Ward J F and Weireich G 1962 Optical rectification Phys. Rev. Lett. 9446

[41] Morris J and Shen Y R 1971 Far-infrared generation by picosecond pulses in electro-optic materials Opt. Commun. 381

[42] Yang K P, Richards P L and Shen Y R 1971 Generation of far-infrared radiation by picosecond light pulses in $\mathrm{LiNbO}_{3}$ Appl. Phys. Lett. 19320

[43] Zhang X-C, Jin Y, Hewitt T D, Sangsiri T, Kingsley L E and Weiner M 1993 Terahertz radiation from electrooptic crystals Ultra-wideband, Short-pulse Electromagnetics ed Bertoni et al (New York: Plenum) p 21

[44] Rice A, Jin Y, Ma X-F, Zhang X-C, Bliss D, Perkin J and Alexander M 1994 Terahertz optical rectification from $\langle 110\rangle$ zincblende crystals Appl. Phys. Lett. 641324

[45] Zhang X-C, Jin Y, Ma X-F, Rice A, Ware K, Bliss D, Perkin J and Alexander M 1994 Sum-frequency generation and difference-frequency generation near bandgap of zincblende crystals Appl. Phys. Lett. 64622

[46] Cook D J and Hochstrasser R M 2000 Intense terahertz pulses by four-wave rectification in air Opt. Lett. 25 1210

[47] Jin Y and Zhang X-C 1995 THz optical rectification Int. J. Nonlinear Opt. Phys. 4459

[48] Carrig T J, Rodriguez G, Sharp Clement T, Taylor A J and Stewart K R 1995 Generation of terahertz radiation using electro-optic crystal mosaics Appl. Phys. Lett. 66 10-2

[49] Carrig T J, Rodriguez G, Sharp Clement T, Taylor A J and Stewart K R 1995 Scaling of terahertz radiation via optical rectification in electro-optic crystals Appl. Phys. Lett. 66 121-3

[50] Cai Y, Brener I, Lopata J, Wynn J, Feiffer L P, Stark J B, Wu Q, Zhang X-C and Federici J F 1998 Coherent terahertz radiation detection: direct comparison between free-space electro-optic sampling and antenna detection Appl. Phys. Lett. 73444

[51] Wu Q and Zhang X-C 1995 Free-space electro-optic sampling of terahertz beam Appl. Phys. Lett. 673523

[52] Nahata A, Auston D H, Heinz T F and Wu C 1996 Coherent detection of freely propagating terahertz radiation by electro-optic sampling Appl. Phys. Lett. 68150

[53] Uhd Jepsen P, Winnewisser C, Schall M, Schya V, Keiding S R and Helm H 1996 Detection of THz pulses by phase retardation in lithium tantalate Phys. Rev. E $\mathbf{5 3} 3052$

[54] Wu Q and Zhang X-C 1996 Electro-optic sampling of freely propagating THz field Opt. Quantum Electron. 28945

[55] Wu Q and Zhang X-C 1996 Ultrafast electro-optic field sensors Appl. Phys. Lett. 681604

[56] Wu Q, Litz M and Zhang X-C 1996 Broadband detection capability of electro-optic field probes Appl. Phys. Lett. $\mathbf{6 8} 2924$

[57] Gallot G and Grischkowsky D 1999 Electro-optic detection of terahertz radiation J. Opt. Soc. Am. B 16 1204-12

[58] Winnewisser C, Uhd Jepsen P, Schall M, Schyja V and Heim H 1997 Electro-optic detection of THz radiation in $\mathrm{LiTaO}_{3}, \mathrm{LiNbO}_{3}$ and ZnTe Appl. Phys. Lett. 703069

[59] Ferguson B 2001 Horizons and hurdles Internal Report Physics Department, Rensselaer Polytechnic Institute

[60] Wynne K and Jaroszynske D A 1998 Superliminal terahertz pulses Opt. Lett. 2425

[61] van der Weide D W 1994 Planar antennas for all-electronic terahertz systems J. Opt. Soc. Am. B $112553-60$

[62] Han P Y and Zhang X-C 1998 Coherent, broadband mid-infrared terahertz beam sensors Appl. Phys. Lett. 73 3049-51

[63] Leitenstorfer A, Hunsche S, Shah J, Nuss M C and Knox W H 1999 Detectors and sources for ultrabroadband electro-optic sampling: experiment and theory Appl. Phys. Lett. 741516

[64] Kawase K et al 2001 Proc. CLEO, CThD1 p 391

[65] Nahata A, Yardley J T and Heinz T 1999 Free space electro-optic detection of continuous-wave terahertz radiation Appl. Phys. Lett. 752524

[66] Pearce J and Mittleman D M 2001 Propagation of single-cycle terahertz pulses in random media Opt. Lett. 26 2002-4 
[67] Markelz A G, Roitberg A and Heilweil E J 2000 Pulsed terahertz spectroscopy of DNA, bovine serum albumin and collagen between 0.1 and $2.0 \mathrm{THz}$ Chem. Phys. Lett. 320 42-8

[68] Brucherseifer M, Nagel M, Bolivar P H, Kurz H, Bosserhoff A and Buttner R 2001 Label-free probing of the binding state of DNA by time-domain terahertz sensing Appl. Phys. Lett. 77 4049-51

[69] Ferguson B, Wang S H, Gray D, Abbott D and Zhang X-C 2002 T-ray diffraction tomography 13th Topical Meeting of Ultrafast Phenomena, ThD5 (Vancouver, Canada)

[70] Woolard D L, Kaul R, Suenram R, Hight Walker A, Globus T and Samuels A 1999 Terahertz electronics for chemical and biological warfare agent detection Proc. IEEE MTT Symp.

[71] Woolard D L et al 1998 Feasibility of submillimeter technology for the identification of biological warfare agents 4th Joint Workshop on Standoff Detection (Williamsburg, VA, 26-28 Oct.)

[72] Walker A R Hight, Suenram R D, Samuels A, Jensen J, Woolard D and Wiebach W 1998 Fourier transform microwave spectroscopy of chemical warfare agents and their synthetic precursors Proc. SPIE 3533 122-7

[73] Woolard D et al 2001 Sensitivity limits and discrimination capability of THz transmission spectroscopy as a technique for biological agent detection Proc. 5th Joint Conf. on Standoff Detection for Chemical and Biological Defense (Williamsburg, VA, 24-28 Sept.) 